TRANSACTIONS OF THE

AMERICAN MATHEMATICAL SOCIETY

Volume 352, Number 2, Pages 823-842

S 0002-9947(99)02273-4

Article electronically published on July 20, 1999

\title{
THE SET OF IDEMPOTENTS \\ IN THE WEAKLY ALMOST PERIODIC COMPACTIFICATION OF THE INTEGERS IS NOT CLOSED
}

\author{
B. BORDBAR AND J. PYM
}

\begin{abstract}
This paper answers negatively the question of whether the sets of idempotents in the weakly almost periodic compactifications of $(\mathbb{N},+)$ and $(\mathbb{Z},+)$ are closed.
\end{abstract}

\section{INTRODUCTION}

The weakly almost periodic compactification $w \mathbb{N}$ of $\mathbb{N}$ is the largest semigroup with a compact topology in which multiplication is continuous in each variable separately and which contains $(\mathbb{N},+)$ as a dense subsemigroup. 'Largest' here means in the sense of universal mapping theorems: any other separately continuous com-

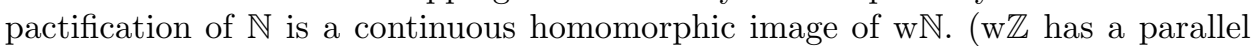
description.) The importance of this compactification was recognised over 30 years ago in the ground-breaking paper of de Leeuw and Glicksberg [6]. In spite of years of study, the subject has been hampered by the difficulty of finding accessible weakly almost periodic functions, that is, functions defined on $\mathbb{N}$ which extend to continuous functions on $w \mathbb{N}$. From one perspective this is not surprising, since weakly almost periodic functions are characterised by the possibility of interchanging two limit processes (see Theorem 2.2 below) and so are intimately connected with one of the fundamental problems of analysis.

Because weakly almost periodic functions are hard to discover, some of the most significant advances have come through indirect methods. This was the case with results about idempotents in $w \mathbb{N}$. (The operation in the commutative semigroup $w \mathbb{N}$ is written + , so that an idempotent $e$ satisfies $e=e+e$.) The question of whether $\mathrm{w} \mathbb{N}$ could contain more than one idempotent was raised by West in connection with a problem in operator theory about the existence of projections, and he found a positive solution in that context [15]. Techniques from harmonic analysis allied with the operator theoretic approach enabled Brown and Moran, in a series of papers, to say much about the lattice structure of the semigroup of idempotents and in particular to show that this semigroup was infinite (see [4]).

However the difficulty of these methods held out little hope of further progress, and indeed no deep new facts about idempotents were discovered for nearly 20 years. Then Ruppert made the important breakthrough: he found a class of weakly almost periodic functions which could be described in elementary terms [14]. His direct

Received by the editors June 16, 1997.

1991 Mathematics Subject Classification. Primary 43A60, 22A15; Secondary 22D05.

Key words and phrases. weakly almost periodic, semigroup compactification, idempotent. 
approach not only allowed him to refine the results of Brown and Moran, but gave new information on the relationship between idempotents and group topologies on $\mathbb{Z}$. His starting point was a very ingenious idea. In the simplest case it involved expanding integers in the form

$$
x=\sum_{i=0}^{\infty} x_{i} k^{i}
$$

where $k \geq 3$ and the digits $x_{i}$ are integers with $-\frac{1}{2} k<x_{i} \leq \frac{1}{2} k$. (His general theory actually uses a variable base in place of the fixed base $k$. The remarkable insight lay in allowing the digits $x_{i}$ to take negative values.) His functions were defined by infinite products taking values in $[0,1]$, and the proof that his functions were weakly almost periodic was hard.

In [2] the first of the present authors presented a theory which was on the same general lines as Ruppert's but which was in some ways simpler. There are two main differences. First the base for the expansion of the integers is taken to be the negative number $(-2)$. This allows the digits to remain positive, and once the simple rules for carrying out addition to a negative base have been worked out, the necessary calculations become easier. Secondly, the interval $[0,1]$ is replaced by $[0, \infty]$. Although this entails handling weakly almost periodic functions which are unbounded, it has the advantage that infinite sums can be considered instead of infinite products. The weakly almost periodic functions which arise have a very simple form (Theorem 2.3 below). We shall, in $\S 3$, give a proof of this result which is even easier than the one in [2].

Ruppert did not solve all the basic problems about idempotents in $w \mathbb{N}$ in [14]. For example, he left open the question of whether the set of idempotents is closed. This appears in the list of problems about semitopological semigroups compiled by Berglund in 1980 [1], and was raised again by Ruppert in his 1984 monograph [12] and his 1990 survey article [13]. That is the question which this paper answers negatively, for both $w \mathbb{N}$ and $w \mathbb{Z}$. To achieve this end, we produce in Theorem 2.8 another new type of weakly almost periodic function, though one which is obtained from a sequence of functions of the form given in [2]. The proof that these functions are weakly almost periodic is technically complicated, and involves (in Lemma 4.3) an elementary result about interchanging the order of limits for a particular kind of double sequence of real numbers. This proof is the subject of $\S 4$. The basic strategy for the whole argument is the same as the one used in [3] to solve the same problem in the simpler situation of the weakly almost periodic compactification of a discrete countable direct sum of finite groups; we present this, the core of our proof, in $\S 2$.

Throughout the paper we shall work with $\mathbb{Z}$ rather than $\mathbb{N}$, as Ruppert did in [14], because $\mathbb{Z}$ arises naturally when expansions to negative bases are considered. The conclusion we desire for $\mathbb{N}$ is an easy consequence of the result for $\mathbb{Z}$ (Corollary 2.10). As usual, $\omega$ denotes the first infinite ordinal (or $\{0\} \cup \mathbb{N}$ ).

\section{The MAIN ARGUMENT}

This section contains the proof of our main result, namely

Theorem 2.1. The set of idempotents in $\mathrm{w} \mathbb{Z}$ is not closed. 
with the exception of the calculations which show that two classes of functions are weakly almost periodic (Theorems 2.3 and 2.8). These arguments will be presented in $\S 3$ and $\S 4$ respectively. We shall deduce the result for $\mathbb{N}$ in Corollary 2.10.

We begin by observing that any integer $x \in \mathbb{Z}$ can be written uniquely in the form

$$
x=\sum_{i \in \omega} x(i)(-2)^{i}=x(0)+x(1) \cdot(-2)+x(2) \cdot(-2)^{2}+\ldots,
$$

where $x(i) \in\{0,1\}$ for each $i$, and $x(i)=1$ for at most finitely many $i$ (this possibility occurs as Example 1.6.1 of [7] and was introduced and used in [10] at the same time as that book appeared). If we define the support of $x, \operatorname{supp} x$, by

$$
\operatorname{supp} x=\{i: x(i) \neq 0\} \text {, }
$$

then $\operatorname{supp} x$ is finite. Obviously $\operatorname{supp} x$ determines $x$. We write $\operatorname{supp} x<k$ if $i<k$ whenever $i \in \operatorname{supp} x$, and $\operatorname{supp} x<\operatorname{supp} y$ if $i<j$ whenever $i \in \operatorname{supp} x$ and $j \in \operatorname{supp} y$. If we have a sequence $\left(x_{n}\right)$ in $\mathbb{Z}$, we say $\operatorname{supp} x_{n} \rightarrow \infty$ when $\min \left(\operatorname{supp} x_{n}\right) \rightarrow \infty$.

Let $c=(c(i))$ be a sequence with $c(i) \in[0, \infty)$ for all $i$. We write

$$
\langle c, x\rangle=\sum_{i \in \omega} c(i) x(i)=\sum_{i \in \operatorname{supp} x} c(i),
$$

and also, for any subset $E$ of $\omega$,

$$
\langle x\rangle_{E}=\sum_{i \in E} c(i) x(i)
$$

In particular, $\langle c, x\rangle_{\emptyset}=0$. Observe too that this function is additive over disjoint sets $E$. Notice that the function $x \mapsto\langle c, x\rangle$ takes values in $[0, \infty)$ and in general is not bounded. We shall therefore need a theory of unbounded weakly almost periodic functions. This is fully described in [2]; we simply quote the main conclusion which we need.

Theorem 2.2. Let $X$ be a compact metrizable space and let $f: \mathbb{Z} \rightarrow X$ be a mapping. Then $f$ has a continuous extension to $\mathrm{w} \mathbb{Z}$ if and only if whenever $\left(x_{m}\right)$, $\left(y_{n}\right)$ are sequences in $\mathbb{Z}$ for which the limits

$$
a=\lim _{m \rightarrow \infty} \lim _{n \rightarrow \infty} f\left(x_{m}+y_{n}\right), \quad b=\lim _{n \rightarrow \infty} \lim _{m \rightarrow \infty} f\left(x_{m}+y_{n}\right)
$$

both exist, then $a=b$.

Of course when $X$ is a compact subset of $\mathbb{C}$, this is just the usual criterion for a bounded complex valued function to be weakly almost periodic; and it is not hard to deduce Theorem 2.2 from that observation using compositions of $f$ with complex-valued functions defined on $X$.

The main result we require about these functions is one of the principal theorems of $[2]$ :

Theorem 2.3. Let $(c(i))_{i \in \omega}$ be a non-increasing sequence of non-negative real numbers with $c(i) \searrow 0$. Then $x \mapsto\langle c, x\rangle$ is weakly almost periodic from $\mathbb{Z}$ to $[0, \infty]$.

We shall give a proof of this result in $\S 3$. In passing, we notice that it is important in this theorem that the range of the function is understood as $[0, \infty]$ and not $[0, \infty)$, because in Theorem 2.2 the target space $X$ has to be compact. (We also remark that the result may not hold for other compactifications of $[0, \infty)$.) 
We next see how functions of the kind described in Theorem 2.3 enable us to detect idempotents. We consider functions $f: \mathbb{Z} \rightarrow([0, \infty],+)$. We call $f$ an oid map if whenever $x, y \in \mathbb{Z}$ and $\operatorname{supp} x<\operatorname{supp} y$ we have $f(x+y)=f(x)+f(y)$. (The term arises from [11]; see also [10]. Ruppert [14] uses the description 'almost homomorphism'.) It is obvious from the last expression in equations $(*)$ that $x \mapsto$ $\langle c, x\rangle$ is an oid map. We also write

$$
H=\bigcap_{r=1}^{\infty} \operatorname{cl}\{x: \operatorname{supp} x \geq r\} .
$$

The following theorem, again from [2], is parallel to a standard result in the theory of the semigroup structure of Stone-Cech compactifications (see [11] or [9]).

Theorem 2.4. $H$ is a compact subsemigroup of $\mathrm{w} \mathbb{Z}$. If $f$ is a weakly almost periodic oid map, then the continuous extension $f: \mathrm{w} \mathbb{Z} \rightarrow([0, \infty],+)$ is a homomorphism on $H$.

Proof. $\{x: \operatorname{supp} x \geq r\}$ is a semigroup, and therefore its closure is, and therefore $H$ is.

Take $x \in \mathbb{Z}$. Let $\zeta \in H$. Let $s=\max (\operatorname{supp} x)$. Then $\zeta \in \operatorname{cl}\{z \in \mathbb{Z}: \operatorname{supp} z \geq s+1\}$. For any such $z$, we have $f(x+z)=f(x)+f(z)$ since $f$ is an oid map, whence letting $z \rightarrow \zeta$ gives $f(x+\zeta)=f(x)+f(\zeta)$. Take $\xi \in H$ and let $x \rightarrow \xi$ to get $f(\xi+\zeta)=f(\xi)+f(\zeta)$, as required.

We shall need a refinement of the last result. We present no more than we shall use. Let $\left(u_{n}\right)$ be a sequence in $\mathbb{Z}$ with $\operatorname{supp} u_{1}<\operatorname{supp} u_{2}<\ldots$ Then it is very easy to see that this sequence has distinct finite sums, meaning that if

$$
u_{i_{1}}+u_{i_{2}}+\cdots+u_{i_{r}}=u_{j_{1}}+u_{j_{2}}+\cdots+u_{j_{s}},
$$

with $i_{1}<i_{2}<\ldots<i_{r}$ and $j_{1}<j_{2}<\ldots<j_{s}$, then $r=s$ and $i_{1}=j_{1}, i_{2}=j_{2}$, $\ldots, i_{r}=j_{r}$. Such sequences play an important role in the theory of Stone-Cech compactifications ([8] or [9]).

Theorem 2.5. Let $\left(u_{n}\right)$ be as above. Then

$$
\begin{aligned}
H\left(u_{n}\right)=\bigcap_{h=0}^{\infty} \bigcap_{k=1}^{\infty} \operatorname{cl}\left\{u_{i_{1}}+u_{i_{2}}+\cdots+u_{i_{r}}: r \in \mathbb{N}, r \geq k,\right. \\
\left.\operatorname{supp} u_{i_{1}} \geq h, \quad \text { and } i_{1}<i_{2}<\ldots<i_{r}\right\}
\end{aligned}
$$

is a subsemigroup of $H$.

Proof. Let $\xi \in H\left(u_{n}\right)$. Then, given any neighbourhood $W$ of $\xi$ and any $h \in \mathbb{N}$, we can find $v \in W$ with $v=u_{i_{1}}+u_{i_{2}}+\cdots+u_{i_{r}}$ (as in the formula which defines $\left.H\left(u_{n}\right)\right)$ such that $\operatorname{supp} v \geq \operatorname{supp} u_{i_{1}} \geq h$. Thus $\xi \in H$. Then

Now let $w=u_{j_{1}}+u_{j_{2}}+\cdots+u_{j_{s}}$ be a second sum of the same kind with $j_{1}>i_{r}$.

$$
v+w=u_{i_{1}}+u_{i_{2}}+\cdots+u_{i_{r}}+u_{j_{1}}+u_{j_{2}}+\cdots+u_{j_{s}}
$$

is also of the same kind. If we take $\xi, \zeta \in H\left(u_{n}\right)$, we can first let $w \rightarrow \zeta$ and then let $v \rightarrow \xi$ to find that $\xi+\zeta \in H\left(u_{n}\right)$.

Next we produce a particular family of functions of the form described in Theorem 2.3. This type of function made a significant appearance in [2]. We first define a strictly increasing sequence $\left(k_{n}\right)$ of integers and a non-increasing sequence 
$\left(\alpha_{n}\right)$ of numbers in $(0,1]$ inductively as follows. We take $k_{0}=1$ and $\alpha_{0}=1$. When $k_{n}$ and $\alpha_{n}$ have been chosen we take $\alpha_{n+1}$ so small that both $\alpha_{n+1} \leq \alpha_{n}$ and $\alpha_{n+1} k_{n} \leq 2^{-(n+1)}$, and then $k_{n+1}$ so large that $\alpha_{n+1}\left(k_{n+1}-k_{n}\right) \geq 1$. (In [2], the place of $\alpha_{n}$ was taken by $2^{-2^{n}}$.) Observe that $\sum_{n} \alpha_{n+1} k_{n} \leq 1$, whereas $\sum_{n \in E} \alpha_{n+1}\left(k_{n+1}-k_{n}\right)=\infty$ for any infinite set $E \subseteq \omega$.

We now define a sequence from which we shall generate a weakly almost periodic function. Take an infinite strictly increasing sequence $\gamma=\left(\gamma_{1}, \gamma_{2}, \ldots\right)$ in $\omega$ with $\gamma_{1} \geq 1$ and $\gamma_{n}+2 \leq \gamma_{n+1}$ for all $n$. Define

$$
d^{\gamma}(i)=\left\{\begin{array}{ll}
\alpha_{\gamma_{1}}, & 0 \leq i \leq k_{\gamma_{1}} \\
\alpha_{\gamma_{n}}, & k_{\gamma_{(n-1)}}<i \leq k_{\gamma_{n}}
\end{array} .\right.
$$

(In reading this paper, it is important to be able to distinguish between $\gamma_{n}-1$ and $\gamma_{n-1}$; to make this easier - especially when these expressions occur as subscripts we shall write the second of these as $\gamma_{(n-1)}$.) Then $\left(d^{\gamma}(i)\right)$ is non-increasing with limit 0 , and so from Theorems 2.3 and 2.4 we obtain immediately

Proposition 2.6. The function

$$
g^{\gamma}(x)=\left\langle d^{\gamma}, x\right\rangle
$$

is weakly almost periodic and is a homomorphism on $H$.

We now define a sequence $\left(u_{n}^{\gamma}\right)_{n=1}^{\infty}$ of integers by writing

$$
u_{n}^{\gamma}(i)= \begin{cases}1 & \text { if } k_{\gamma_{n}-1}<i \leq k_{\gamma_{n}} \\ 0 & \text { otherwise }\end{cases}
$$

and putting $u_{n}^{\gamma}=\sum_{i \in \omega} u_{n}^{\gamma}(i)(-2)^{i}$. Note that if $m<n$ then $\operatorname{supp} u_{m}^{\gamma}<\operatorname{supp} u_{n}^{\gamma}$. Then

$$
\begin{aligned}
g^{\gamma}\left(u_{n}^{\gamma}\right) & =\sum\left\{d^{\gamma}(i): u_{n}^{\gamma}(i)=1\right\}=\sum\left\{\alpha_{\gamma_{n}}: k_{\gamma_{n}-1}<i \leq k_{\gamma_{n}}\right\} \\
& =\alpha_{\gamma_{n}}\left(k_{\gamma_{n}}-k_{\gamma_{n}-1}\right) \geq 1 .
\end{aligned}
$$

Since $g^{\gamma}$ is an oid map, if $n_{1}<n_{2}<\ldots<n_{r}$ we have

$$
g^{\gamma}\left(u_{n_{1}}^{\gamma}+\cdots+u_{n_{r}}^{\gamma}\right)=g^{\gamma}\left(u_{n_{1}}^{\gamma}\right)+\cdots+g^{\gamma}\left(u_{n_{r}}^{\gamma}\right) \geq r
$$

from which we see that $g^{\gamma}$ takes the value $\infty$ on the whole of the semigroup $H\left(u_{n}^{\gamma}\right)$ of Theorem 2.5. In particular, if $e^{\gamma}$ is any idempotent in $H\left(u_{n}^{\gamma}\right)$ we have $g^{\gamma}\left(e^{\gamma}\right)=\infty$.

Now take a second sequence $\eta=\left(\eta_{1}, \eta_{2}, \ldots\right)$ of the same form as $\gamma$, but suppose that $\eta$ and $\gamma$ are disjoint - no element of $\omega$ is in both. Then, for any integer $n$, since $\left(k_{n}\right)$ is strictly increasing, there is a unique integer $m=m(n)$ for which $k_{\eta_{(m-1)}}<$ $k_{\gamma_{n}} \leq k_{\eta_{m}-1}$. Notice that as $n \rightarrow \infty$ we have $m(n) \rightarrow \infty$. Since $\gamma_{n} \leq \eta_{m}-1$, we have also $\alpha_{\eta_{m}} \leq \alpha_{\gamma_{n}+1}$. Thus we find that

$$
g^{\eta}\left(u_{n}^{\gamma}\right)=\sum\left\{d^{\gamma}(i): u_{n}^{\gamma}(i)=1\right\}=\alpha_{\eta_{m}}\left(k_{\gamma_{n}}-k_{\gamma_{n}-1}\right) \leq \alpha_{\gamma_{n}+1} k_{\gamma_{n}} \leq 2^{-\left(\gamma_{n}+1\right)} .
$$

The integers $\gamma_{n}$ are distinct for distinct values of $n$, so if $n_{1}<n_{2}<\ldots<n_{r}$ and $m=m\left(n_{1}\right)$ we find that

$$
g^{\eta}\left(u_{n_{1}}^{\gamma}+\cdots+u_{n_{r}}^{\gamma}\right)=g^{\eta}\left(u_{n_{1}}^{\gamma}\right)+\cdots+g^{\eta}\left(u_{n_{r}}^{\gamma}\right) \leq \sum_{n \geq n_{1}} 2^{-\left(\gamma_{n}+1\right)}=2^{-\gamma_{n_{1}}} .
$$

Whatever $r$ does, when $n_{1} \rightarrow \infty, \gamma_{n_{1}} \rightarrow \infty$, so that $g^{\eta}\left(H\left(u_{n}^{\gamma}\right)\right)=0$. In particular, $g^{\eta}\left(e^{\gamma}\right)=0$.

Of course it is easy to find a countable set $\Gamma$ of disjoint subsequences $\gamma$ in $\omega$. Thus we have proved: 
Lemma 2.7. For any countable set $\Gamma$ of disjoint sequences $\gamma$ in $\omega$ there is a family $\left\{e^{\gamma}: \gamma \in \Gamma\right\}$ of distinct idempotents in $H$ such that for $\eta, \gamma \in \Gamma$

$$
g^{\eta}\left(e^{\gamma}\right)=\left\{\begin{aligned}
\infty & \text { if } \eta=\gamma \\
0 & \text { if } \eta \neq \gamma
\end{aligned}\right.
$$

We shall go on to prove that if $\zeta$ is any cluster point of the family $\left(e^{\gamma}\right)$ in $\mathrm{w} \mathbb{Z}$ then $\zeta$ is not an idempotent (indeed, $\zeta$ generates a semigroup isomorphic with $(\mathbb{N},+)$ ). To achieve this we need a weakly almost periodic function which will distinguish between elements of the form $e^{\gamma}$ and elements of the form $e^{\eta}+e^{\gamma}$ (when $\eta \neq \gamma$ ). Unfortunately some simple choices of functions do not work. For example, the function $\sum_{\theta \in \Gamma} g^{\theta}$ fails because it takes the value $\infty$ on all these elements. The function defined in the next theorem is satisfactory, but it is difficult to prove that it is weakly almost periodic (this we shall do in $\S 4$ ). We first define, for any continuous function $f: \mathrm{w} \mathbb{Z} \rightarrow[0, \infty]$,

$$
f \wedge 1(\xi)=\min \{f(\xi), 1\} \quad(\xi \in \mathrm{w} \mathbb{Z}) .
$$

This is continuous, and so its restriction to $\mathbb{Z}$ (which we again denote by $f \wedge 1$ ) is weakly almost periodic.

Theorem 2.8. Let $\Gamma$ be a countable set of disjoint sequences in $\omega$. Then the function

$$
G=\sum_{\gamma \in \Gamma}\left(g^{\gamma} \wedge 1\right)
$$

is weakly almost periodic from $\mathbb{Z}$ to $[0, \infty]$.

We shall now derive Theorem 2.1 from Theorem 2.8. Let $\zeta$ be any cluster point of $\left(e^{\gamma}\right)$. From Lemma 2.7 we see that for $\eta, \gamma \in \Gamma$ we have $g^{\eta} \wedge 1\left(e^{\gamma}\right)$ is 0 if $\eta \neq \gamma$ and is 1 if $\eta=\gamma$. Therefore $G\left(e^{\gamma}\right)=1$ for every $\gamma$. Since $G$, being a weakly almost periodic function, is continuous on $\mathrm{w} \mathbb{Z}$, we see that $G(\zeta)=1$.

Now take $\eta \neq \gamma$. Because $g^{\gamma}$ is a homomorphism on $H$ we find that $g^{\gamma}\left(e^{\eta}+e^{\gamma}\right)=$ $g^{\gamma}\left(e^{\eta}\right)+g^{\gamma}\left(e^{\gamma}\right)=\infty$, and similarly $g^{\eta}\left(e^{\eta}+e^{\gamma}\right)=\infty$, while if $\theta \neq \eta$ and $\theta \neq \gamma$ we have $g^{\theta}\left(e^{\eta}+e^{\gamma}\right)=0$. Therefore $G\left(e^{\eta}+e^{\gamma}\right)=2$. Letting first $e^{\gamma} \rightarrow \zeta$ and then $e^{\eta} \rightarrow \zeta$, we find that $G(\zeta+\zeta)=2$.

Therefore $\zeta \neq \zeta+\zeta$, so that $\zeta$ is not idempotent.

Of course, the method of the above proof will also establish that if $\gamma_{1}, \ldots, \gamma_{k}$ are distinct elements of $\Gamma$, then $G\left(e^{\gamma_{1}}+\cdots+e^{\gamma_{k}}\right)=k$, whence $G(\zeta+\cdots+\zeta)=k$. This shows that $\zeta, \zeta+\zeta, \zeta+\zeta+\zeta, \ldots$ are all distinct:

Theorem 2.9. Any cluster point of the set $\left\{e^{\gamma}: \gamma \in \Gamma\right\}$ of idempotents generates an algebraic copy of $(\mathbb{N},+)$ in $\mathrm{w} \mathbb{Z}$.

Thus there are at least $\mathfrak{c}$ copies of $\mathbb{N}$ in $w \mathbb{Z}$ (since there is a family of $\mathfrak{c}$ almost disjoint subsets of the countable set $\Gamma$ ). Whether the closure of any of these sets is isomorphic with $\mathrm{w} \mathbb{N}$ we do not know.

Corollary 2.10. The set of idempotents in $\mathrm{w} \mathbb{N}$ is not closed.

Proof. We give two proofs. (i) The first requires little knowledge about $\mathrm{w} \mathbb{N}$. There are two natural homomorphisms $\phi, \psi: \mathbb{N} \rightarrow \mathbb{Z}$, given by $\phi(n)=n$ and $\psi(n)=-n$.

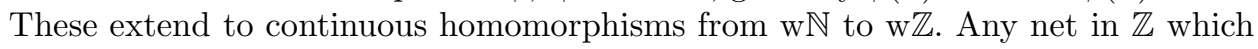


does not converge to the isolated point 0 either has a subnet which is contained in $\phi(\mathbb{N})$ or one in $\psi(\mathbb{N})$, and since $\phi(\mathrm{w} \mathbb{N})$ and $\psi(\mathrm{w} \mathbb{N})$ are compact and so closed, we deduce that $\mathrm{w} \mathbb{Z} \backslash\{0\}=\phi(\mathrm{w} \mathbb{N}) \cup \psi(\mathrm{w} \mathbb{N})$. Because $\phi$ and $\psi$ are homomorphisms and $\mathrm{w} \mathbb{N}$ is compact, the idempotents in $\phi(\mathrm{w} \mathbb{N})(\operatorname{resp} \psi(\mathrm{w} \mathbb{N}))$ are precisely the images under $\phi(\operatorname{resp} \psi)$ of the idempotents in $w \mathbb{N}$, so that if $E(w \mathbb{N})$ is the set of idempotents in $\mathrm{w} \mathbb{N}$ we have that $\phi(E(\mathrm{w} \mathbb{N})) \cup \psi(E(\mathrm{w} \mathbb{N}))$ is $E(\mathrm{w} \mathbb{Z}) \backslash\{0\}$. If $E(\mathrm{w} \mathbb{N})$ were closed we would deduce that $E($ w $\mathbb{Z})$ was compact, which we now know is not true.

(ii) Experts in the subject will know of Ruppert's remarkable result in Theorem 4.2.1(i) of [13]: the continuous map $\phi$ above is algebraically an isomorphism of $E(\mathrm{w} \mathbb{N})$ onto $E(\mathrm{w} \mathbb{Z}) \backslash\{0\}$. Therefore if $E(\mathrm{w} \mathbb{N})$ is compact, so is $E(\mathrm{w} \mathbb{Z})$.

\section{The Proof of Theorem 2.3}

In this section, we prove Theorem 2.3. The method is the same as in [2], but the details are simpler. In $\S 4$ we shall need the inequalities obtained here. The proof depends on the peculiarities of the arithmetic of addition in $\mathbb{Z}$ when numbers are expressed to the base $(-2)$. When $x=\sum_{i=0}^{\infty} x(i)(-2)^{i}, y=\sum_{i=0}^{\infty} y(i)(-2)^{i}$ and $x+y=\sum_{i=0}^{\infty} z(i)(-2)^{i}$, we shall write

$$
\begin{aligned}
& x=x(0) \quad x(1) \quad x(2) \quad \ldots
\end{aligned}
$$

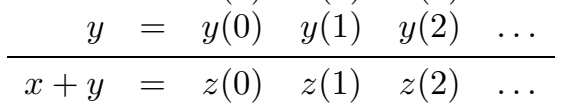

Expressed in this way, the calculation

$$
\text { 1. }(-2)^{n}+1 \cdot(-2)^{n}=(-1) \cdot(-2)^{n+1}=(1+(-2)) \cdot(-2)^{n+1}=(-2)^{n+1}+(-2)^{n+2} \text {, }
$$

becomes

$$
\begin{array}{lllllll}
\ldots & 0 & 1 & 0 & 0 & 0 & \ldots \\
\ldots & 0 & 1 & 0 & 0 & 0 & \ldots \\
\hline \ldots & 0 & 0 & 1 & 1 & 0 & \ldots
\end{array}
$$

where all the other digits are zero. Put in the language of elementary arithmetic, if the sum in the $n^{\text {th }}$ column is 2 , then $(-1)$ should be carried into the $(n+1)^{\text {st }}$ column, and that is the same as carrying 1 into the $(n+1)^{\text {st }}$ column and also 1 into the $(n+2)^{\text {nd }}$. Thus we also see that

$$
\begin{array}{llllll}
\ldots & 0 & 1 & 1 & 0 & \ldots \\
\ldots & 0 & 1 & 0 & 0 & \ldots \\
\hline \ldots & 0 & 0 & 0 & 0 & \ldots
\end{array}
$$

In fact, carrying into the $r^{\text {th }}$ column for any $r$ must be either $(-1)$ or 1 , and the latter happens only when, as in the situation just described, $(-1)$ has been carried into the $(r-1)^{\text {st }}$ column and the two digits in the $(r-1)^{\text {st }}$ column are both 0 . (For more details about arithmetic in negative bases, see [5].)

We now come to our first inequality. Its proof is very elementary, but is complicated by the number of cases which have to be considered.

Lemma 3.1. Let $c(i) \searrow 0$. Let $x, y \in \mathbb{Z}$, and let $r, s \in \omega$ with $r<s$. Suppose $y(i)=0$ for $r \leq i<s$. Then 
(i) if there is no carrying into the $r^{\text {th }}$ place,

$$
\left|\langle c, x+y\rangle_{[r, s)}-\langle c, x\rangle_{[r, s)}-\langle c, y\rangle_{[r, s)}\right|=0
$$

(ii) in all cases,

$$
\left|\langle c, x+y\rangle_{[r, s)}-\langle c, x\rangle_{[r, s)}-\langle c, y\rangle_{[r, s)}\right| \leq 2 c(r) .
$$

Proof. First observe that $\langle c, y\rangle_{[r, s)}=0$ (this term is included in the formula only because it will be needed later). The situation we have is this:

$$
\begin{array}{ccccccccccc}
\ldots & x(r-1) & x(r) & x(r+1) & \ldots & x(p) & x(p+1) & \ldots & x(s-1) & x(s) & \ldots \\
\ldots & y(r-1) & 0 & 0 & \ldots & 0 & 0 & \ldots & 0 & y(s) & \ldots \\
\hline \ldots & z(r-1) & z(r) & z(r+1) & \ldots & z(p) & z(p+1) & \ldots & z(s-1) & z(s) & \ldots
\end{array}
$$

(i) If there is no carrying from the $(r-1)^{\text {st }}$ place into the $r^{\text {th }}$ place, then there is no carrying at all between the $r^{\text {th }}$ and $s^{\text {th }}$ places, so that $z(i)=x(i)$ for $r \leq i<s$. Thus

$$
\left|\langle c, x+y\rangle_{[r, s)}-\langle c, x\rangle_{[r, s)}\right|=0 .
$$

(ii) Since $0 \leq 2 c(r)$, (ii) holds in the circumstances of (i). We may therefore suppose that there is carrying into the $r^{\text {th }}$ place. We know that what is carried must be either $(-1)$ or 1 .

CASE (a). Suppose that $(-1)$ is carried into the $r^{\text {th }}$ place, and that there is carrying as far as into the $p^{\text {th }}$ place, but nothing is carried into the $(p+1)^{\text {st }}$ place. We shall first take $r+1<p<s$. Then there will be carrying into the $(r+1)^{\text {st }}$ place; because this does not happen if $x(r)=1$, we must have $x(r)=0$. Then $z(r)=1$, and 1 is carried into the $(r+1)^{\text {st }}$ place. As there is carrying into the $(r+2)^{\text {nd }}$ place, $x(r+1)=0$ is not possible. Thus $x(r+1)=1$, and then $z(r+1)=0$ and $(-1)$ is carried into the $(r+2)^{\text {nd }}$ place. This process repeats itself until the $p^{\text {th }}$ place is reached. There are now two possible patterns. Firstly, when $p=r+2 k$ for some positive integer $k$, we find

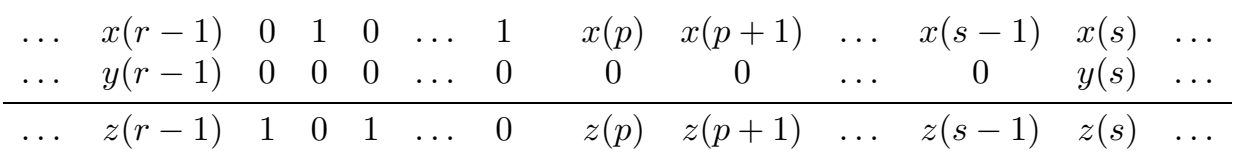

and when $p=r+(2 k+1)$ for some $k$, we have

$$
\begin{aligned}
& \begin{array}{llllllllllllll}
\ldots & x(r-1) & 0 & 1 & 0 & \ldots & 0 & x(p) & x(p+1) & \ldots & x(s-1) & x(s) & \ldots
\end{array} \\
& \begin{array}{ccccccccccccc}
\ldots & y(r-1) & 0 & 0 & 0 & \ldots & 0 & 0 & 0 & \ldots & 0 & y(s) & \ldots \\
\ldots & z(r-1) & 1 & 0 & 1 & \ldots & 1 & z(p) & z(p+1) & \ldots & z(s-1) & z(s) & \ldots
\end{array}
\end{aligned}
$$

In the first situation, when $p=r+2 k$, we have that $(-1)$ is carried into the $p^{\text {th }}$ place, and since carrying stops at the $p^{\text {th }}$ place by assumption, $x(p)$ must be 1 , and then $z(p)=0$. Since there is no carrying thereafter, $z(i)=x(i)$ for $p+1 \leq i<s$. 
Thus (using the hypothesis that $(c(i))$ is a decreasing sequence)

$$
\begin{aligned}
\langle c, x+y\rangle_{[r, s)}-\langle c, x\rangle_{[r, s)} & \\
& =c(r)-c(r+1)+c(r+2)-c(r+3)+\cdots-c(r+2 k-1)-c(r+2 k) \\
& =(c(r)-c(r+1))+(c(r+2)-c(r+3))+ \\
& \quad \cdots+(c(r+2 k-2)-c(r+2 k-1))-c(r+2 k) \\
& \geq-c(r+2 k),
\end{aligned}
$$

and on the other hand

$$
\begin{aligned}
& \langle c, x+y\rangle_{[r, s)}-\langle c, x\rangle_{[r, s)} \\
& =c(r)-(c(r+1)-c(r+2))- \\
& \quad \cdots-(c(r+2 k-3)-c(r+2 k-2))-c(r+2 k-1)-c(r+2 k) \\
& \quad \leq c(r) .
\end{aligned}
$$

Therefore

$$
\left|\langle c, x+y\rangle_{[r, s)}-\langle c, x\rangle_{[r, s)}\right| \leq \max \{c(r), c(r+2 k)\}=c(r) .
$$

Thus the conclusion of the lemma holds (even with $c(r)$ in place of $2 c(r)$ ).

When $p=r+2 k+1$ we have that 1 is carried into the $p^{\text {th }}$ place, and because there is no further carrying, $x(p)=0$ and $z(p)=1$. Again $z(i)=x(i)$ for $p+1 \leq i<s$. Thus

$$
\begin{aligned}
\langle c, x+y\rangle_{[r, s)}-\langle c, x\rangle_{[r, s)} & =c(r)-c(r+1)+\cdots+c(r+2 k)+c(r+2 k+1) \\
& \geq c(r+2 k+1)>0,
\end{aligned}
$$

and on the other hand,

$$
\begin{aligned}
& \langle c, x+y\rangle_{[r, s)}-\langle c, x\rangle_{[r, s)} \\
& \quad \leq c(r)-(c(r+1)-c(r+2))-\cdots-(c(r+2 k-1)-c(r+2 k))+c(r+2 k+1) \\
& \quad \leq c(r)+c(r+2 k+1) \leq 2 c(r) .
\end{aligned}
$$

So the conclusion of the lemma holds in this case too.

There are some minor cases to deal with before Case (a) is complete. Above we took $p>r+1$. When $p=r+1$ the result turns out to be just like the case $p=r+2 k+1$ with $k=0$, as it should. When $p=r$ the situation is

$$
\begin{array}{cccccccc}
\ldots & x(r-1) & 1 & x(r+1) & \ldots & x(s-1) & x(s) & \ldots \\
\ldots & y(r-1) & 0 & 0 & \ldots & 0 & y(s) & \ldots \\
\hline \ldots & z(r-1) & 0 & x(r+1) & \ldots & x(s-1) & z(s) & \ldots
\end{array}
$$

so that

$$
\left|\langle c, x+y\rangle_{[r, s)}-\langle c, x\rangle_{[r, s)}\right|=|-c(r)|=c(r) .
$$


Finally we must confront the possibility that $p=s$ - that is, that carrying goes right through to the $s^{\text {th }}$ place. Again there are two cases, when $p=r+2 k$ and when $p=r+2 k+1$. In the former case, the situation is

$$
\begin{array}{lllllllll}
\ldots & x(r-1) & 0 & 1 & 0 & \ldots & 1 & x(s) & \ldots \\
\ldots & y(r-1) & 0 & 0 & 0 & \ldots & 0 & y(s) & \ldots \\
\hline \ldots & z(r-1) & 1 & 0 & 1 & \ldots & 0 & z(s) & \ldots
\end{array}
$$

and so

$$
\langle c, x+y\rangle_{[r, s)}-\langle c, x\rangle_{[r, s)}=c(r)-c(r+1)+\cdots+c(r+2 k-2)-c(r+2 k)
$$

which is positive and does not exceed $c(r)$. If $p=2 k+1$, we find in a similar way that the difference is again positive and less than $2 c(r)$. Case (a) is finished.

CASE (b). When 1 is carried into the $r^{\text {th }}$ place, we can argue as in Case (a) to obtain the same conclusion. We leave the details to the reader.

Next we shall derive an inequality like the one in Lemma 3.1 but without the restriction on $y$. Note that for any $i$, because each of $x(i), y(i)$ and $(x+y)(i)$ is either 0 or 1 we have

$$
|c(i)(x+y)(i)-c(i) x(i)-c(i) y(i)| \leq 2 c(i) .
$$

In particular, if $y(i) \neq 0$, so that $y(i)=1$, the right hand side here is just $2 c(i) y(i)$.

Now take any integers $r, s$ with $r<s$. Write $\{i: r \leq i \leq s$ and $y(i) \neq 0\}$ as an ordered set of elements $r \leq r_{0} \leq r_{1} \leq \ldots \leq r_{\ell} \leq s$. Then on each interval $\left[r_{j-1}, r_{j}\right)$ and also on $\left[r_{\ell}, s\right]$ we have, using $(* *)$ and Lemma 3.1,

$$
\begin{aligned}
& \left|\langle c, x+y\rangle_{\left[r_{j-1}, r_{j}\right)}-\langle c, x\rangle_{\left[r_{j-1}, r_{j}\right)}-\langle c, y\rangle_{\left[r_{j-1}, r_{j}\right)}\right| \\
& \leq\left|\langle c, x+y\rangle_{\left\{r_{j-1}\right\}}-\langle c, x\rangle_{\left\{r_{j-1}\right\}}-\langle c, y\rangle_{\left\{r_{j-1}\right\}}\right| \\
& \quad+\left|\langle c, x+y\rangle_{\left[r_{j-1}+1, r_{j}\right)}-\langle c, x\rangle_{\left[r_{j-1}+1, r_{j}\right)}-\langle c, y\rangle_{\left[r_{j-1}+1, r_{j}\right)}\right| \\
& \leq 2 c\left(r_{j-1}\right) y\left(r_{j-1}\right)+2 c\left(r_{j-1}+1\right) \leq 4 c\left(r_{j-1}\right) y\left(r_{j-1}\right)=4\langle c, y\rangle_{\left[r_{j-1}, r_{j}\right)}
\end{aligned}
$$

because $y\left(r_{j-1}\right)=1$ and $y(i)=0$ on $\left[r_{j-1}+1, r_{j}\right)$.

To deal with the interval $\left[r, r_{0}\right)$ if $r_{0}>r$, notice that when there is no carrying into the $r^{\text {th }}$ place then from Lemma 3.1 (i) we find that

$$
\left|\langle c, x+y\rangle_{\left[r, r_{0}\right)}-\langle c, x\rangle_{\left[r, r_{0}\right)}-\langle c, y\rangle_{\left[r, r_{0}\right)}\right|=0 \leq 4\langle c, y\rangle_{\left[r, r_{0}\right)},
$$

while if there is carrying into the $r^{\text {th }}$ place then Lemma 3.1 (ii) applies to $\left[r, r_{0}\right)$. If we add these results over the disjoint intervals $\left[r, r_{0}\right),\left[r_{0}, r_{1}\right), \ldots,\left[r_{\ell-1}, r_{\ell}\right),\left[r_{\ell}, s\right]$ we obtain the following conclusions for the case in which $s<\infty$ :

(i) if either $y(r) \neq 0$ or there is no carrying into the $r^{\text {th }}$ place, then

$$
\left|\langle c, x+y\rangle_{[r, s)}-\langle c, x\rangle_{[r, s)}-\langle c, y\rangle_{[r, s)}\right| \leq 4\langle c, y\rangle_{[r, s)} ;
$$

(ii) in all cases,

$$
\left|\langle c, x+y\rangle_{[r, s)}-\langle c, x\rangle_{[r, s)}-\langle c, y\rangle_{[r, s)}\right| \leq 2 c(r)+4\langle c, y\rangle_{[r, s)} .
$$

Naturally there are corresponding inequalities with the roles of $x$ and $y$ interchanged. Putting these together, we obtain the following lemma in the case $s<\infty$ :

Lemma 3.2. Let $0 \leq r \leq s \leq \infty$. Then

(i) if there is no carrying into the $r^{\text {th }}$ place (and so in particular if $r=0$ ), then

$$
\left|\langle c, x+y\rangle_{[r, s)}-\langle c, x\rangle_{[r, s)}-\langle c, y\rangle_{[r, s)}\right| \leq 4 \min \left\{\langle c, x\rangle_{[r, s)},\langle c, y\rangle_{[r, s)}\right\} ;
$$


(ii) in all cases,

$$
\left|\langle c, x+y\rangle_{[r, s)}-\langle c, x\rangle_{[r, s)}-\langle c, y\rangle_{[r, s)}\right| \leq 2 c(r)+4 \min \left\{\langle c, x\rangle_{[r, s)},\langle c, y\rangle_{[r, s)}\right\} .
$$

Proof. We have only to prove the result when $s=\infty$. This is trivial, for $x, y$ and $x+y$ have supports which are finite, and so the case $s=\infty$ is the same as $s=s_{0}$ for any sufficiently large $s_{0}$.

Remarks on subsequences 3.3. We need to make use of some complex arguments involving subsequences. We shall try to simplify things by presenting the basic ideas here. We are concerned with expressions of the form

$$
a=\lim _{n} \lim _{m} f\left(x_{m}+y_{n}\right), \quad b=\lim _{m} \lim _{n} f\left(x_{m}+y_{n}\right) .
$$

If we substitute subsequences $\left(x_{m_{r}}\right),\left(y_{n_{s}}\right)$ for $\left(x_{m}\right),\left(y_{n}\right)$, these limits still exist and remain unchanged. Thus if we are trying to prove that $a=b$ we can work with any subsequences which have desirable properties. For simplicity of notation (which can rapidly get very complicated) we shall usually simply assume that our original sequences have these properties.

One particular problem which arises frequently is that we want to ensure that another countable family of limits, say $\lim _{m} \phi(m, n)$ and $\lim _{n} \phi(m, n)$, exist. If $\phi$ takes its values in a compact metric space, we can certainly find a subset $M_{1}$ of $\mathbb{N}$ such that $\lim _{m \in M_{1}} \phi(m, 1)$ exists. Then we find $M_{2} \subseteq M_{1}$ such that $\lim _{m \in M_{2}} \phi(m, 2)$ exists, and so on. We take the set $M^{\prime}$ to consist of the first element of $M_{1}$, the second of $M_{2}$, and so on. With this diagonal construction all the $\operatorname{limits}_{m \in M^{\prime}} \phi(m, n)$ exist. We can then take a second subset $N^{\prime}$ by operating with the $n$ variable, and we find that the family $\left\{\phi(m, n): m \in M^{\prime}, n \in N^{\prime}\right\}$ satisfies our requirements.

Now suppose that the terms of the double sequence $(\phi(m, n))$ have a property $A$ when $m>n$ and a property $B$ when $n>m$. We want to take subsequences such that the limits in the last paragraph exist but also such that these properties are retained. The family we arrived at there is not adequate, since the sets $M^{\prime}$ and $N^{\prime}$ may be different. What we must do is first construct the family $\{\phi(m, n)$ : $\left.m \in M^{\prime}, n \in M^{\prime}\right\}$; this family has the property $A$ when $m>n$ and $B$ when $n>m$, as well as all the $m$-limits existing. Now we find in the same manner a subset $N^{\prime \prime} \subseteq M^{\prime}$ for which the $n$-limits of the new family exist, and finally $\left\{\phi(m, n): m \in N^{\prime \prime}, n \in N^{\prime \prime}\right\}$ satisfies all the requirements.

We are now in a position to prove Theorem 2.3, that if $c(i) \searrow 0$ then $x \mapsto\langle c, x\rangle$ is a weakly almost periodic mapping into $[0, \infty]$. We shall show that the iterated limit condition of Theorem 2.2 is satisfied. Let $\left(x_{m}\right),\left(y_{n}\right)$ be two sequences for which the iterated $\operatorname{limits}_{\lim _{m}} \lim _{n}\left\langle c, x_{m}+y_{n}\right\rangle$ and $\lim _{n} \lim _{m}\left\langle c, x_{m}+y_{n}\right\rangle$ exist in $[0, \infty]$. Our first step is to replace these sequences by subsequences (which we continue to denote by $\left(x_{m}\right)$ and $\left.\left(y_{n}\right)\right)$ for which $a=\lim _{m}\left\langle c, x_{m}\right\rangle$ and $b=\lim _{n}\left\langle c, y_{n}\right\rangle$ both exist in $[0, \infty]$; the values of the iterated limits remain unchanged.

Now we use a diagonal process to put these sequences into a canonical form. The first coordinates $\left(x_{m}(1)\right)$ form a sequence in the two-element space $\{0,1\}$. This therefore has a constant subsequence, say with all terms equal to $u(1)$. That sequence has a further subsequence whose second term is constant (equal to $u(2)$, say), and so on. We take a diagonal sequence, and denote it by $\left(x_{m}\right)$ again. This $\left(x_{m}\right)$ has the form

$$
x_{m}=\left(u(1), u(2), \ldots, u(m), x_{m}(m+1), \ldots, x_{m}\left(M_{m}\right), 0,0, \ldots\right)
$$


where $M_{m}=\max \left\{i: x_{m}(i) \neq 0\right\}$. Similarly we can suppose

$$
y_{n}=\left(v(1), v(2), \ldots, v(n), y_{n}(n+1), \ldots, y_{n}\left(N_{n}\right), 0,0, \ldots\right)
$$

with $N_{n}=\max \left\{i: y_{n}(i) \neq 0\right\}$.

Let us write $u=(u(1), u(2), \ldots), v=(v(1), v(2), \ldots)$ and $w=u+v(w$ is defined by doing the arithmetic formally, but the process works); observe that if $r \leq \min \{m, n\}$ then $w(r)=\left(x_{m}+y_{n}\right)(r)$. Also $\langle c, w\rangle_{[r, s)}$ is defined whenever $0 \leq r \leq s \leq \infty$, and is finite if $s<\infty$.

CASE (i). Suppose $a<\infty$ and $b<\infty$. We shall show that the two iterated limits are the same. Since they converge to finite limits, $\left(\left\langle c, x_{m}\right\rangle\right)$ and $\left(\left\langle c, y_{n}\right\rangle\right)$ are bounded sequences of real numbers. Now from Lemma 3.2(i) (with $r=0$ ) we get

$$
\left|\left\langle c, x_{m}+y_{n}\right\rangle-\left\langle c, x_{m}\right\rangle-\left\langle c, y_{n}\right\rangle\right| \leq 4 \min \left\{\left\langle c, x_{m}\right\rangle,\left\langle c, y_{n}\right\rangle\right\}
$$

so that all the iterated limits of $\left\langle c, x_{m}+y_{n}\right\rangle$ are finite. We now take $n>M_{m}$. We split the range $[0, \infty)$ into three parts.

(1) On $[0, m]$ we have $\left\langle c, x_{m}+y_{n}\right\rangle_{[0, m]}=\langle c, w\rangle_{[0, m]}$, since $w(r)=\left(x_{m}+y_{n}\right)(r)$ for $r \leq \min \{m, n\}$.

(2) On $[m+1, n]$ we have $y_{n}(i)=v(i)$. Therefore

$$
\langle c, v\rangle_{[m+1, n]}=\left\langle c, y_{n}\right\rangle_{[m+1, n]} \leq\left\langle c, y_{n}\right\rangle_{[m+1, \infty)} \leq\left\langle c, y_{n}\right\rangle,
$$

so that

$$
\langle c, v\rangle_{[m+1, \infty)}=\lim _{n}\langle c, v\rangle_{[m+1, n]} \leq \lim _{n}\left\langle c, y_{n}\right\rangle=b<\infty
$$

Hence, since $\langle c, v\rangle$ here stands for a convergent series,

$$
\lim _{m} \lim _{n}\left\langle c, y_{n}\right\rangle_{[m+1, n]}=\lim _{m}\langle c, v\rangle_{[m+1, \infty)}=0 .
$$

Also, since $y_{n}(i)=v(i)$ for $i \leq n,\left\langle c, x_{m}+y_{n}\right\rangle_{[m+1, n]}=\left\langle c, x_{m}+v\right\rangle_{[m+1, n]}$, so that for each $m, \lim _{n}\left\langle c, x_{m}+y_{n}\right\rangle_{[m+1, n]}$ exists in $[0, \infty]$. Now from Lemma 3.2(ii)

$$
\begin{aligned}
\mid\left\langle c, x_{m}+y_{n}\right\rangle_{[m+1, n]} & -\left\langle c, x_{m}\right\rangle_{[m+1, n]}-\left\langle c, y_{n}\right\rangle_{[m+1, n]} \mid \\
& =\left|\left\langle c, x_{m}+v\right\rangle_{[m+1, n]}-\left\langle c, x_{m}\right\rangle_{[m+1, n]}-\langle c, v\rangle_{[m+1, n]}\right| \\
& \leq 2 c(m+1)+4\langle c, v\rangle_{[m+1, n]} .
\end{aligned}
$$

Take limits over $n$ and then over $m$, to find

$$
\left|\lim _{m} \lim _{n}\left\langle c, x_{m}+y_{n}\right\rangle_{[m+1, n)}-\lim _{m}\left\langle c, x_{m}\right\rangle_{[m+1, \infty)}-0\right| \leq 0+0,
$$

so that

$$
\lim _{m} \lim _{n}\left\langle c, x_{m}+y_{n}\right\rangle_{[m+1, n)}=\lim _{m}\left\langle c, x_{m}\right\rangle_{[m+1, \infty)}
$$

(3) On $[n+1, \infty)$ we have from Lemma 3.2(ii)

$$
\begin{aligned}
\mid\left\langle c, x_{m}+y_{n}\right\rangle_{[n+1, \infty)}-\left\langle c, x_{m}\right\rangle_{[n+1, \infty)} & -\left\langle c, y_{n}\right\rangle_{[n+1, \infty)} \mid \\
& \leq 2 c(n+1)+4\left\langle c, x_{m}\right\rangle_{[n+1, \infty)} .
\end{aligned}
$$

Since $n>M_{m}, x_{m}(i)=0$ on $[n+1, \infty)$, whence the middle term on the left hand side of this inequality disappears and the right hand side is just $2 c(n+1)$. Thus

$$
\lim _{m} \lim _{n}\left\langle c, x_{m}+y_{n}\right\rangle_{[n+1, \infty)}=\lim _{n}\left\langle c, y_{n}\right\rangle_{[n+1, \infty)} .
$$

Putting these three parts together, we find that

$$
\lim _{m} \lim _{n}\left\langle c, x_{m}+y_{n}\right\rangle=\lim _{m}\langle c, w\rangle_{[0, m]}+\lim _{m}\left\langle c, x_{m}\right\rangle_{[m+1, \infty)}+\lim _{n}\left\langle c, y_{n}\right\rangle_{[n+1, \infty)} .
$$


Symmetry now shows that the other iterated limit has the same value. This case is finished.

CAse (ii). At least one of $a, b$ is infinite - say $a=\infty$. From Lemma 3.2(i)

$$
\left|\left\langle c, x_{m}+y_{n}\right\rangle-\left\langle c, x_{m}\right\rangle-\left\langle c, y_{n}\right\rangle\right| \leq 4\left\langle c, y_{n}\right\rangle,
$$

which shows that, for each fixed $n, \lim _{m}\left\langle c, x_{m}+y_{n}\right\rangle=\infty$, because $\lim _{m}\left\langle c, x_{m}\right\rangle=$ $a=\infty$ and all other terms in the inequality are finite. Therefore,

$$
\lim _{n} \lim _{m}\left\langle c, x_{m}+y_{n}\right\rangle=\infty \text {. }
$$

If also $b=\infty$, then symmetry allows us to conclude that both iterated limits are $\infty$. If $b$ is finite, we can take the $n$-limit in the inequality to get

$$
\left|\lim _{n}\left\langle c, x_{m}+y_{n}\right\rangle-\left\langle c, x_{m}\right\rangle-b\right| \leq 4 b,
$$

and then as above we find that $\lim _{m} \lim _{n}\left\langle c, x_{m}+y_{n}\right\rangle=\infty$.

Since the iterated limits are equal, our function is weakly almost periodic and Theorem 2.3 is proved.

\section{The PRoof of Theorem 2.8}

In this section, we prove Theorem 2.8. We begin with a few elementary lemmas.

Lemma 4.1. (i) If $a, b, c \geq 0$ then $|a-b| \leq c$ implies $|a \wedge 1-b \wedge 1| \leq c \wedge 1$.

(ii) Let $a, b, c, \ell \geq 0$ and let $k \geq 1$ be an integer. Suppose that $|a-b-c| \leq \ell+k b$. Then $|a \wedge 1-b \wedge 1-c \wedge 1| \leq \ell+k(b \wedge 1)$.

Proof. (i) For non-negative numbers $a, b, c$ it is obvious that $a \leq b+c$ implies $a \wedge 1 \leq b \wedge 1+c \wedge 1$, and our conclusion follows easily.

(ii) We have both

$$
\begin{aligned}
& a \leq \ell+k b+b+c \\
& \quad \Longrightarrow a \wedge 1 \leq(\ell+k b+b+c) \wedge 1 \leq \ell \wedge 1+k(b \wedge 1)+b \wedge 1+c \wedge 1
\end{aligned}
$$

and

$$
\begin{aligned}
b+c \leq \ell+k b+a & \Longrightarrow c \leq \ell+(k-1) b+a \\
& \Longrightarrow c \wedge 1 \leq \ell \wedge 1+(k-1)(b \wedge 1)+a \wedge 1,
\end{aligned}
$$

whence the result follows (and we could even have $\ell \wedge 1$ in place of $\ell$ on the right).

Our second lemma is just a tool for the third.

Lemma 4.2. For each $\gamma \in \omega$ let $\left(a_{m}^{\gamma}\right)_{m \in \omega},\left(b_{n}^{\gamma}\right)_{n \in \omega}$ be sequences satisfying

(i) $0 \leq a_{m}^{\gamma}, b_{n}^{\gamma} \leq 1$,

(ii) there is a constant $K$ such that $\sum_{\gamma \in \omega}\left(a_{m}^{\gamma}+b_{n}^{\gamma}\right) \leq K$ for all $m, n$.

Let $(\gamma(m, n))$ be a double sequence with values in $\omega$ such that $\lim _{n} \lim _{m} \gamma(m, n)=$ $\infty$. Then, provided the iterated limit exists, $\lim _{n} \lim _{m}\left(a_{m}^{\gamma(m, n)}+b_{n}^{\gamma(m, n)}\right) \leq 1$.

Proof. Suppose not, so that for some $\epsilon>0$

$$
\lim _{n} \lim _{m}\left(a_{m}^{\gamma(m, n)}+b_{n}^{\gamma(m, n)}\right)=1+2 \epsilon .
$$

Then there is $N$ and for each $n>N$ there is $M_{n}$ such that when $n>N$ and $m>M_{n}$ we have

$$
a_{m}^{\gamma(m, n)}+b_{n}^{\gamma(m, n)}>1+\epsilon
$$


Hence for such $n$ and $m$ we have

$$
a_{m}^{\gamma(m, n)}>1+\epsilon-b_{n}^{\gamma(m, n)} \geq \epsilon,
$$

and similarly $b_{n}^{\gamma(m, n)}>\epsilon$.

Take an integer $r$ with $r \epsilon>K$. Suppose that for some $n>N$ we have $\gamma(m, n) \underset{m}{\longrightarrow}$ $\infty$. Then we can choose $m_{1}, \ldots, m_{r}>M_{n}$ with $\gamma\left(m_{1}, n\right), \ldots, \gamma\left(m_{r}, n\right)$ all distinct, so that

$$
r \epsilon<b_{n}^{\gamma\left(m_{1}, n\right)}+\cdots+b_{n}^{\gamma\left(m_{r}, n\right)} \leq \sum_{\gamma} b_{n}^{\gamma} \leq K,
$$

a contradiction.

Since our hypotheses guarantee that our limits exist, the alternative is that the sequences $(\gamma(m, n))_{m=1}^{\infty}$ converge to a finite limit for each $n$, and since they are integer-valued, there must be an integer, $\gamma^{\prime}(n)$ say, such that $\gamma(m, n)=\gamma^{\prime}(n)$ eventually, say for $m>M_{n}^{\prime}$. Again from our hypotheses, $\gamma^{\prime}(n) \rightarrow \infty$. We take $n_{1}, \ldots, n_{r}$ all greater than $N$ with $\gamma^{\prime}\left(n_{1}\right), \ldots, \gamma^{\prime}\left(n_{r}\right)$ all distinct. Take also $m>$ $\max \left\{M_{n_{1}}, \ldots, M_{n_{r}}, M_{n_{1}}^{\prime}, \ldots, M_{n_{r}}^{\prime}\right\}$. Then $a_{m}^{\gamma\left(m, n_{j}\right)}>\epsilon$ for $j=1, \ldots, r$, and we obtain a contradiction as before.

Lemma 4.3. For each $\gamma \in \omega$ let $\left(a_{m}^{\gamma}\right)_{m \in \omega},\left(b_{n}^{\gamma}\right)_{n \in \omega}$ be sequences of non-negative numbers for which there is a constant $K$ such that $\sum_{\gamma \in \omega}\left(a_{m}^{\gamma} \wedge 1+b_{n}^{\gamma} \wedge 1\right) \leq K$ for all $m, n$. Then, provided all the limits exist $($ in $[0, \infty]$, but here they are necessarily finite)

$$
\lim _{n} \lim _{m} \sum_{\gamma \in \omega}\left(\left(a_{m}^{\gamma}+b_{n}^{\gamma}\right) \wedge 1\right)=\lim _{m} \lim _{n} \sum_{\gamma \in \omega}\left(\left(a_{m}^{\gamma}+b_{n}^{\gamma}\right) \wedge 1\right) .
$$

Proof. Because $(a+b) \wedge 1=(a \wedge 1+b \wedge 1) \wedge 1$ for any non-negative numbers $a$ and $b$, we may replace $a_{m}^{\gamma}$ by $a_{m}^{\gamma} \wedge 1$ and $b_{n}^{\gamma}$ by $b_{n}^{\gamma} \wedge 1$ throughout the statement of the lemma, or in other words we may assume that $0 \leq a_{m}^{\gamma}, b_{n}^{\gamma} \leq 1$ for all $\gamma, m, n$.

Now assume that all the limits in the lemma exist. By passing to subsequences we may assume that $\lim _{m} a_{m}^{\gamma}$ and $\lim _{n} b_{n}^{\gamma}$ also exist for every $\gamma$.

For a given pair $m, n$ we put

$$
E(m, n)=\left\{\gamma: a_{m}^{\gamma}+b_{n}^{\gamma}>1\right\}
$$

and we also write

$$
E=\left\{\gamma: \lim _{m} a_{m}^{\gamma}+\lim _{n} b_{n}^{\gamma}>1\right\} .
$$

Now obviously $\operatorname{card} E(m, n) \leq K$. Thus the sequences $(\operatorname{card} E(m, n))_{m=1}^{\infty}$ for each $n$ and $(\operatorname{card} E(m, n))_{n=1}^{\infty}$ for each $m$ are bounded sequences of integers, and so have constant subsequences. Following Remarks 3.3, we may assume that $(E(m, n))$ has the properties that $\operatorname{card} E(m, n)$ is constant (say equal to $r_{n}$ ) when $m>n$, and constant and equal to $s_{m}$ when $n>m$. But now $\left(r_{n}\right)$ is a sequence of integers bounded by $K$ and so also has a constant subsequence, say with value $r$. Thus by taking further subsequences we may assume that $\operatorname{card} E(m, n)=r$ when $m>n$ and $\operatorname{card} E(m, n)=s$ when $n>m$.

When $m>n$ we may now write

$$
E(m, n)=\left\{\gamma_{1}(m, n), \gamma_{2}(m, n), \ldots, \gamma_{r}(m, n)\right\} .
$$


By passing to subsequences again (with Remarks 3.3 in mind) we may assume that $\lim _{n} \lim _{m} \gamma_{j}(m, n)$ exists in $\omega \cup\{\infty\} \subseteq[0, \infty]$ for $1 \leq j \leq r$. We now need two cases. If $\lim _{n} \lim _{m} \gamma_{j}(m, n)=\infty$, then we may apply Lemma 4.2 to conclude that

$$
\lim _{n} \lim _{m}\left(a_{m}^{\gamma_{j}(m, n)}+b_{n}^{\gamma_{j}(m, n)}-1\right) \leq 0
$$

and since $\gamma_{j}(m, n) \in E(m, n)$ we see that the terms in this limit are all positive, so that the limit itself must be zero. The alternative is that there is an integer $\gamma_{j}$ such that $\lim _{n} \lim _{m} \gamma_{j}(m, n)=\gamma_{j}$, in which case there is $N_{0}$ and for each $n>N_{0}$ an $M_{n}^{*}$ such that when $n>N_{0}$ and $m>M_{n}^{*}$ we have $\gamma_{j}(m, n)=\gamma_{j}$, so that also

$$
a_{m}^{\gamma_{j}(m, n)}+b_{n}^{\gamma_{j}(m, n)}=a_{m}^{\gamma_{j}}+b_{n}^{\gamma_{j}} .
$$

Therefore

$$
\lim _{n} \lim _{m}\left(a_{m}^{\gamma_{j}(m, n)}+b_{n}^{\gamma_{j}(m, n)}\right)=\lim _{n} \lim _{m}\left(a_{m}^{\gamma_{j}}+b_{n}^{\gamma_{j}}\right)=\lim _{m} a_{m}^{\gamma_{j}}+\lim _{n} b_{n}^{\gamma_{j}} .
$$

This limit is $>1$ if $\gamma_{j} \in E$ by definition of $E$, but otherwise is 1 .

Now to calculate the iterated limit of

$$
\sum_{\gamma \in \omega}\left(a_{m}^{\gamma}+b_{n}^{\gamma}\right) \wedge 1=\sum_{\gamma \in \omega} a_{m}^{\gamma}+\sum_{\gamma \in \omega} b_{n}^{\gamma}-\sum_{\gamma \in E(m, n)}\left(a_{m}^{\gamma}+b_{n}^{\gamma}-1\right)
$$

in the case in which the $m$-limit is taken first, we use

$$
\sum_{\gamma \in E(m, n)}\left(a_{m}^{\gamma}+b_{n}^{\gamma}-1\right)=\sum_{j=1}^{r}\left(a_{m}^{\gamma_{j}(m, n)}+b_{n}^{\gamma_{j}(m, n)}-1\right),
$$

and we see that the only nonzero terms here arise from $\sum_{\gamma \in E}\left(a_{m}^{\gamma}+b_{n}^{\gamma}-1\right)$. Conversely, it is easy to see that elements of $E$ give rise to nonzero terms in this limit. Thus we have

$$
\begin{aligned}
\lim _{n} \lim _{m} \sum_{\gamma \in \omega}\left(a_{m}^{\gamma}+b_{n}^{\gamma}\right) \wedge 1 & =\lim _{m} \sum_{\gamma \in \omega} a_{m}^{\gamma}+\lim _{n} \sum_{\gamma \in \omega} b_{n}^{\gamma}-\lim _{n} \lim _{m} \sum_{\gamma \in E(m, n)}\left(a_{m}^{\gamma}+b_{n}^{\gamma}-1\right) \\
& =\lim _{m} \sum_{\gamma \in \omega} a_{m}^{\gamma}+\lim _{n} \sum_{\gamma \in \omega} b_{n}^{\gamma}-\sum_{\gamma \in E}\left(\lim _{m} a_{m}^{\gamma}+\lim _{n} b_{n}^{\gamma}-1\right) .
\end{aligned}
$$

The symmetry of this expression shows that the iterated limit taken in the other order must be the same. Our proof is finished.

We now begin to deal with the function $G$ defined in Theorem 2.8. Let $\Gamma=$ $\left(\gamma^{1}, \gamma^{2}, \ldots\right)$ be a countable collection of disjoint sequences. We write

$$
G_{h}=\sum_{r=1}^{h}\left(g^{\gamma^{r}} \wedge 1\right),
$$

so that $G(x)=\lim _{h} G_{h}(x)$ for each $x$. We wish to prove $G$ is weakly almost periodic. To this end we take sequences $\left(x_{m}\right),\left(y_{n}\right)$ in $\mathbb{Z}$ for which the iterated limits of

$$
G\left(x_{m}+y_{n}\right)
$$

exist in $[0, \infty]$. By passing to subsequences (see 3.3) we can assume that the limits of $\left(G\left(x_{m}\right)\right)$ and $\left(G\left(y_{n}\right)\right)$ exist in $[0, \infty]$, and that all the iterated limits of

$$
g^{\gamma} \wedge 1\left(x_{m}+y_{n}\right)
$$


exist for each $\gamma \in \Gamma$. Note that each $g^{\gamma} \wedge 1$ is weakly almost periodic (see just before Theorem 2.8) and - since finite sums of weakly almost periodic functions are weakly almost periodic $-G_{h}$ is weakly almost periodic for any $h$.

We now make some further modifications to our sequences and define some new ones. First we use the methods of $\S 3$ to write our sequences $\left(x_{m}\right),\left(y_{n}\right)$ in the forms

$$
\begin{aligned}
& x_{m}=\left(u(0), u(1), \quad \ldots, \quad u(m), \quad x_{m}(m+1), \quad \ldots, \quad x_{m}\left(M_{m}\right), \quad 0, \quad 0, \quad \ldots\right), \\
& y_{n}=\left(v(0), \quad v(1), \quad \ldots, \quad v(n), \quad y_{n}(n+1), \quad \ldots, \quad y_{n}\left(N_{n}\right), \quad 0, \quad 0, \quad \ldots\right) \text {, }
\end{aligned}
$$

where $M_{m}=\max \left\{i: x_{m}(i) \neq 0\right\}$ and $N_{n}=\max \left\{i: y_{n}(i) \neq 0\right\}$. We put

$$
\begin{aligned}
& u_{m}=(u(0), u(1), \ldots, u(m), \quad 0,0, \ldots), \\
& v_{n}=(v(0), \quad v(1), \quad \ldots, \quad v(n), \quad 0, \quad 0, \quad \ldots),
\end{aligned}
$$

and write

$$
\begin{aligned}
& u=(u(0), \quad u(1), \quad u(2), \quad \ldots), \\
& v=(v(0), \quad v(1), \quad v(2), \quad \ldots) .
\end{aligned}
$$

We put $w=u+v$ where the addition is carried out formally; but observe that if we put

$$
w_{r}=(w(0), \quad w(1), \quad \ldots, \quad w(r), \quad 0, \quad 0, \quad \ldots),
$$

then if $r \leq \min \{m, n\}$ for $0 \leq i \leq r$ we have

$$
w(i)=w_{r}(i)=\left(u_{m}+v_{n}\right)(i)=\left(x_{m}+y_{n}\right)(i) .
$$

Finally we write

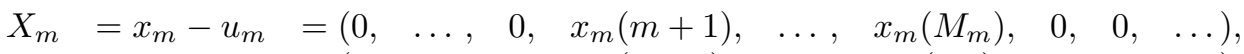

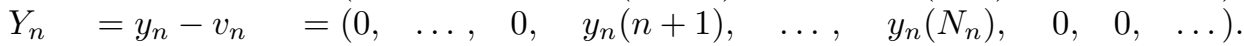

We write $g_{[r, s)}^{\gamma}(x)=\left\langle d^{\gamma}, x\right\rangle_{[r, s)}$ and $G_{[r, s)}=\sum_{\gamma} g_{[r, s)}^{\gamma} \wedge 1$.

Lemma 4.4. For any $x, y$ and $0 \leq r<s \leq \infty$ we have

$$
\left|G_{[r, s)}(x+y)-G_{[r, s)}(x)-G_{[r, s)}(y)\right| \leq 2 D(r)+4 \min \left\{G_{[r, s)}(x), G_{[r, s)}(y)\right\},
$$

where $D(r)=0$ if $r=0$ and $D(r) \rightarrow 0$ as $r \rightarrow \infty$.

Proof. First take $r>0$. From Lemma 3.2(ii), for $r<s \leq \infty$, any $\gamma$ and any $x, y$,

$$
\left|g_{[r, s)}^{\gamma}(x+y)-g_{[r, s)}^{\gamma}(x)-g_{[r, s)}^{\gamma}(y)\right| \leq 2 d^{\gamma}(r)+4 g_{[r, s)}^{\gamma}(x) .
$$

We apply Lemma 4.1(ii) to get

$$
\left|g_{[r, s)}^{\gamma} \wedge 1(x+y)-g_{[r, s)}^{\gamma} \wedge 1(x)-g_{[r, s)}^{\gamma} \wedge 1(y)\right| \leq 2 d^{\gamma}(r)+4 g_{[r, s)}^{\gamma} \wedge 1(x),
$$

and then sum over $\gamma$, putting $D(r)=\sum_{\gamma} d^{\gamma}(r)$, to find that

$$
\left|G_{[r, s)}(x+y)-G_{[r, s)}(x)-G_{[r, s)}(y)\right| \leq 2 D(r)+4 G_{[r, s)}(x) .
$$

Taking into account the symmetry between $x$ and $y$, this will prove our result when $r>0$ if we show that $D(r) \rightarrow 0$. Now from the definition of $d^{\gamma}(r)$ just before Proposition 2.6 we see that $d^{\gamma}(r)=\alpha_{\gamma_{n(r)}}$ where $\gamma_{n(r)}$ is the unique term in the sequence $\gamma=\left(\gamma_{n}\right)$ for which $k_{\gamma_{(n-1)}}<r \leq k_{\gamma_{n}}$. Now for any $r$, there is precisely one integer $p=p(r)$ for which $k_{p-1}<r \leq k_{p}$. Notice that $p(r) \rightarrow \infty$ as $r \rightarrow \infty$. Since $\left(k_{n}\right)$ is strictly increasing, this implies that $p(r) \leq \gamma_{n(r)}$ for each $r$. Thus

$$
D(r)=\sum_{\gamma} \alpha_{\gamma_{n(r)}} \leq \sum_{\gamma} 2^{-\gamma_{n(r)}}
$$


since, by definition of $\alpha_{n}$, we have $\alpha_{n} \leq \alpha_{n} k_{n-1} \leq 2^{-n}$. Now $\left(\gamma_{n(r)}\right)_{\gamma \in \Gamma}$ is a sequence of distinct integers each $\geq p(r)$. Thus $D(r) \leq 2^{-p(r)-1} \rightarrow 0$ as $r \rightarrow \infty$.

For the case in which $r=0$ we have only to use Lemma 3.2(i) in place of 3.2(ii) to see that we can here replace $d^{\gamma}(r)$ and then $D(r)$ by zero.

If, in the preceeding proof, we sum only over a subset of $\Gamma$ we get a similar inequality (and we can use the same term $2 D(r)$, though strictly speaking it is larger than necessary):

Corollary 4.5. Under the conditions of the lemma,

$$
\begin{aligned}
\mid\left(G-G_{h}\right)_{[r, s)}\left(x_{m}+y_{n}\right) & -\left(G-G_{h}\right)_{[r, s)}\left(x_{m}\right)-\left(G-G_{h}\right)_{[r, s)}\left(y_{n}\right) \mid \\
\leq & 2 D(r)+4 \min \left\{\left(G-G_{h}\right)_{[r, s)}\left(x_{m}\right),\left(G-G_{h}\right)_{[r, s)}\left(y_{n}\right)\right\} .
\end{aligned}
$$

The first case in which we establish that the iterated limit condition holds for $G$ is easy. The proof is virtually the same as that given at the end of $\S 3$ for Case (ii) of Theorem 2.3.

Case (i). Suppose that $G\left(y_{n}\right) \rightarrow \infty$ as $n \rightarrow \infty$. Then with $r=0, s=\infty$ in Lemma 4.4 we have

$$
\left|G\left(x_{m}+y_{n}\right)-G\left(x_{m}\right)-G\left(y_{n}\right)\right| \leq 4 G\left(x_{m}\right) .
$$

For each fixed $m$ we find that $\lim _{n} G\left(x_{m}+y_{n}\right)=\infty$, since all the other terms in the inequality except $G\left(y_{n}\right)$ remain finite as $n \rightarrow \infty$. Thus $\lim _{m} \lim _{n} G\left(x_{m}+y_{n}\right)=\infty$.

If also $G\left(x_{m}\right) \rightarrow \infty$, the equality of the iterated limits follows from symmetry. Otherwise $G\left(x_{m}\right) \rightarrow \ell<\infty$ (say). Then, taking the $m$-limit in the above inequality we find that

$$
\left|\lim _{m} G\left(x_{m}+y_{n}\right)-\ell-G\left(y_{n}\right)\right| \leq 4 \ell .
$$

Since $G\left(y_{n}\right) \rightarrow \infty$, this implies that $\lim _{n} \lim _{m} G\left(x_{m}+y_{n}\right)=\infty$.

The case in which $G\left(x_{m}\right) \rightarrow \infty$ follows from symmetry.

CASE (ii). Let both $\lim _{m} G\left(x_{m}\right)$ and $\lim _{n} G\left(y_{n}\right)$ be finite. Then also $G(u)$ (defined to be $\lim _{m} G_{[0, m)}(u)=\lim _{m} G_{[0, m)}\left(x_{m}\right)$ ) exists and is finite, because $\left(G_{[0, m)}(u)\right)$ is an increasing sequence and $G_{[0, m)}(u) \leq G\left(x_{m}\right)$ for each $m$. Similarly, for each $n, G_{[0, n)}(v) \leq G(v)<\infty$. Also, from

$$
\left|G_{[0, m)}(u+v)-G_{[0, m)}(u)-G_{[0, m)}(v)\right| \leq 4 G_{[0, m)}(u)
$$

(Lemma 4.4) we find that

$$
G_{[0, m)}(w) \leq 5 G_{[0, m)}(u)+G_{[0, m)}(v) \leq 5 G(u)+G(v)<\infty,
$$

so that $G(w)$ is finite.

Lemma 4.6. For each $\epsilon>0$ there is $h_{0}$ such that $0 \leq\left(G-G_{h}\right)(u)<\epsilon, 0 \leq$ $\left(G-G_{h}\right)(v)<\epsilon$, and $0 \leq\left(G-G_{h}\right)(w)<\epsilon$ when $h>h_{0}$.

Proof. We have $\left(G_{h}\right)_{[0, m)}(u) \nearrow_{h} G_{[0, m)}(u) \nearrow_{m} G(u)<\infty$. Since $\left(G_{h}\right)_{[0, m)}(u) \leq$ $G_{h}(u) \leq G(u)$, we easily deduce the result for $u$, and the results for $v, w$ are obtained in the same way.

Since $G_{h}$ is weakly almost periodic, its iterated limits are equal, and we need to investigate the iterated limits of $G-G_{h}$. We fix the $\epsilon$ and $h_{0}$ of Lemma 4.6.

Lemma 4.7. For each integer $M$ there is $n \geq M$ such that $v(n)=v(n+1)=0$. 
Proof. Recall that for any $\gamma$ and $n$ we have $\alpha_{\gamma_{n}}\left(k_{\gamma_{n}}-k_{\gamma_{n}-1}\right) \geq 1$, and so if $x \in \mathbb{Z}$ satisfies $x(i)=1$ for $k_{\gamma_{n}-1}<i \leq k_{\gamma_{n}}$, then $g^{\gamma}(x) \geq 1$. Now all the coordinates $d^{\gamma}(i)$ for $k_{\gamma_{n}-1}<i \leq k_{\gamma_{n}}$ are equal to $\alpha_{\gamma_{n}}$, so that if there are never two consecutive zero digits of $v$ in this range, then at least $\frac{1}{2}\left(k_{\gamma_{n}}-k_{\gamma_{n}-1}\right)-1$ of these digits are 1 and hence $g_{\left(k_{\gamma_{n}-1}, k_{\gamma_{n}}\right]}^{\gamma}(v) \geq \frac{1}{2}-\alpha_{\gamma_{n}}$. Since there are infinitely many $\gamma_{n}$ with $k_{\gamma_{n}-1} \geq M$, we find that $g_{[M, \infty)}^{\gamma}(v)=\infty$. Therefore $g_{[M, \infty)}^{\gamma} \wedge 1(v)=1$. This is for every $\gamma$, so that $G(v)=\infty$, contrary to our assumptions.

We now define $J(m)$ (more precisely $J_{v}(m)$, but our $v$ is fixed) to be the smallest integer exceeding $m+2$ for which $v(J(m)-2)=v(J(m)-1)=0$. The point here is that if an integer $x$ also has $x(J(m)-2)=x(J(m)-1)=0$, then in calculating the sum $x+v$ there is no carrying into the $J(m)^{\text {th }}$ place; carrying in base $(-2)$ arithmetic is either 1 (affecting the next place) or -1 (affecting at most the next two places). The precise situation in which we shall wish to apply this observation is as follows. Recall that $y_{n}(i)=v(i)$ for $i \leq n$.

Lemma 4.8. Let $m$ be an integer. Take $n>J(m)$. Suppose $\operatorname{supp} x \leq m$. Then $\left(x+y_{n}\right)(i)=y_{n}(i)$ for $i \geq J(m)$ and so for $i>n$.

Now we consider

$$
g^{\gamma}\left(x_{m}+y_{n}\right)-g^{\gamma}\left(X_{m}\right)-g^{\gamma}\left(Y_{n}\right),
$$

where $\gamma$ is any element of $\Gamma$. We take some $m$ and fix it temporarily. We take $n$ to be large - at least $n>J\left(M_{m}\right)$. We write $g^{\gamma}$ as $g_{[0, m]}^{\gamma}+g_{(m, n]}^{\gamma}+g_{(n, \infty)}^{\gamma}$. First, on $[0, m]$ we have $x_{m}+y_{n}=w$, so that

$$
g_{[0, m]}^{\gamma}\left(x_{m}+y_{n}\right)=g_{[0, m]}^{\gamma}(w) .
$$

The definition of $X_{m}$ shows that $g^{\gamma}\left(X_{m}\right)=g_{\left(m, M_{m}\right]}^{\gamma}\left(X_{m}\right)=g_{(m, n]}^{\gamma}\left(X_{m}\right)$, since $n>M_{m}$ and $x_{m}$ is zero on $\left(M_{m}, n\right]$. Also on $(0, n]$ we have $y_{n}=v$. Therefore, applying Lemma 3.2(ii) to $g^{\gamma}$,

$$
\begin{aligned}
\mid g_{(m, n]}^{\gamma}\left(x_{m}\right. & \left.+y_{n}\right)-g_{(m, n]}^{\gamma}\left(X_{m}\right)-g_{(m, n]}^{\gamma}(v) \mid \\
& =\left|g_{(m, n]}^{\gamma}\left(x_{m}+v\right)-g_{(m, n]}^{\gamma}\left(x_{m}\right)-g_{(m, n]}^{\gamma}(v)\right| \leq 2 d^{\gamma}(m)+4 g_{(m, n]}^{\gamma}(v),
\end{aligned}
$$

whence

$$
\left|g_{(m, n]}^{\gamma}\left(x_{m}+v\right)-g_{(m, n]}^{\gamma}\left(X_{m}\right)\right| \leq 2 d^{\gamma}(m)+5 g_{(m, n]}^{\gamma}(v) .
$$

On $(n, \infty)$ from Lemma 4.8 we see that $g_{(n, \infty)}^{\gamma}\left(x_{m}+y_{n}\right)=g_{(n, \infty)}^{\gamma}\left(y_{n}\right)$, and the definition of $Y_{n}$ shows that $g_{(n, \infty)}^{\gamma}\left(y_{n}\right)=g^{\gamma}\left(Y_{n}\right)$, so that

$$
g_{(n, \infty)}^{\gamma}\left(x_{m}+y_{n}\right)-g^{\gamma}\left(Y_{n}\right)=0 .
$$

Putting these three facts together gives

$$
\left|g^{\gamma}\left(x_{m}+y_{n}\right)-g^{\gamma}\left(X_{m}\right)-g^{\gamma}\left(Y_{n}\right)\right| \leq g_{[0, m]}^{\gamma}(w)+2 d^{\gamma}(m)+5 g_{(m, n]}^{\gamma}(v) .
$$

Therefore, using Lemma 4.1(i),

$$
\begin{aligned}
\mid g^{\gamma} \wedge 1\left(x_{m}+y_{n}\right) & -\left(g^{\gamma}\left(X_{m}\right)+g^{\gamma}\left(Y_{n}\right)\right) \wedge 1 \mid \\
\leq & \left(2 d^{\gamma}(m)+g_{[0, m]}^{\gamma}(w)+5 g_{(m, n]}^{\gamma}(v)\right) \wedge 1 \\
\leq & 2 d^{\gamma}(m)+g^{\gamma} \wedge 1(w)+5 g^{\gamma} \wedge 1(v) .
\end{aligned}
$$


Replacing $\gamma$ by $\gamma^{r}$, summing from $h+1$ to $\infty$, where $h>h_{0}$, and using Lemma 4.6 gives

$$
\begin{aligned}
\mid\left(G-G_{h}\right)\left(x_{m}+y_{n}\right) & -\sum_{r=h+1}^{\infty}\left(g^{\gamma^{r}}\left(X_{m}\right)+g^{\gamma^{r}}\left(Y_{n}\right)\right) \wedge 1 \mid \\
& \leq 2 D(m)+\left(G-G_{h}\right)(w)+5\left(G-G_{h}\right)(v) \leq 2 D(m)+6 \epsilon .
\end{aligned}
$$

Now we have assumed that the iterated limits of $G\left(x_{m}+y_{n}\right)$ exist and we have arranged for the iterated limits of $G_{h}\left(x_{m}+y_{n}\right)$ to exist. If we pass to subsequences $\left(m_{s}\right)$ and $\left(n_{t}\right)$, these limits remain unchanged. We choose $\left(m_{s}\right)$ and $\left(n_{t}\right)$ so that the iterated limits (in both orders) of

$$
\sum_{r=h+1}^{\infty}\left(g^{\gamma^{r}}\left(X_{m_{s}}\right)+g^{\gamma^{r}}\left(Y_{n_{t}}\right)\right) \wedge 1
$$

exist. Then, since $D(m) \rightarrow 0$ as $m \rightarrow \infty$, we find that

$$
\left|\lim _{m} \lim _{n}\left(G-G_{h}\right)\left(x_{m}+y_{n}\right)-\lim _{s} \lim _{t} \sum_{r=h+1}^{\infty}\left(g^{\gamma^{r}}\left(X_{m_{s}}\right)+g^{\gamma^{r}}\left(Y_{n_{t}}\right)\right) \wedge 1\right| \leq 6 \epsilon .
$$

We can now deduce from Lemma 4.3 that the second of these iterated limits coincides with the limit taken in the other order. (We substitute $g^{\gamma^{r}}\left(X_{m_{s}}\right)$ for the $a_{m}^{\gamma}$ of that lemma, and $g^{\gamma^{r}}\left(Y_{n_{t}}\right)$ for $b_{n}^{\gamma}$. We have

$$
\sum_{r=h+1}^{\infty} g^{\gamma^{r}}\left(X_{m_{s}}\right) \wedge 1 \leq \sum_{\gamma \in \Gamma} g^{\gamma}\left(x_{m_{s}}\right) \wedge 1 \leq G\left(x_{m_{s}}\right) .
$$

In the case we are considering, the sequence $\left(G\left(x_{m_{s}}\right)\right)$ is bounded because it is convergent. The same argument shows that $\left(G\left(y_{n_{t}}\right)\right)$ is bounded. Thus the hypotheses of the lemma are satisfied.) Hence

$$
\left|\lim _{m} \lim _{n}\left(G-G_{h}\right)\left(x_{m}+y_{n}\right)-\lim _{n} \lim _{m}\left(G-G_{h}\right)\left(x_{m}+y_{n}\right)\right| \leq 12 \epsilon,
$$

and since the iterated limits of $G_{h}\left(x_{m}+y_{n}\right)$ coincide, we conclude that the iterated limits of $G\left(x_{m}+y_{n}\right)$ must be equal.

Thus $G$ is weakly almost periodic, and our proof is complete.

\section{REFERENCES}

1. J F Berglund, Problems about semitopological semigroups, Semigroup Forum 16 (1980), 373383. MR 81f:20098

2. B Bordbar, Weakly almost periodic functions on $\mathbb{N}$ with a negative base, J London Math Soc (to appear).

3. B Bordbar and J Pym, The weakly almost periodic compactification of a direct sum of finite groups, Math Proc Cambridge Phil Soc 124 (1998), 421-449. CMP 98:16

4. G Brown and W Moran, The idempotent semigroups of compact monothetic semigroups, Proc. Roy. Irish Acad., Sect. A , 72 (1972), 17-33. MR 46:3682

5. T Budak, N Işık and J Pym, Subsemigroups of Stone-Čech compactifications, Math. Proc. Camb. Phil. Soc. 99 (1994), 116-137. MR 94c:54067

6. K de Leeuw and I Glicksberg, Applications of almost periodic compactifications, Acta Math 105 (1961), 63-97. MR 24:A1632

7. G A Edgar, Measure, Topology and Fractal Geometry, Springer, Berlin, 1990. MR 92a:54001

8. N Hindman, The ideal structure of the space of $\kappa$-uniform ultrafilters on a discrete semigroup, Rocky Mountain Math J 16 (1986), 685-701. MR 88d:54031 
9. N Hindman and D Strauss, Algebra in the Stone-Čech Compactification, de Gruyter, Berlin, 1998.

10. T Papazyan, Oids, finite sums and the structure of the Stone-Čech compactification of a discrete semigroup, Semigroup Forum 42 (1991), 265-277. MR 92b:54051

11. J S Pym, Semigroup structure in Stone-Čech compactifications, J. London Math. Soc. 36 (1987), 421-428. MR 89b:54043

12. W A F Ruppert, Compact Semitopological Semigroups: An Intrinsic Theory, Lecture Notes in Mathematics 1079, Springer, Berlin, 1984. MR 86e:22001

13. W A F Ruppert, Compact semitopological semigroups, The Analytical and Topological Theory of Semigroups (K H Hofmann, J D Lawson and J S Pym, eds.), de Gruyter, Berlin, 1990, pp. 133-170. MR 91i:22006

14. W A F Ruppert, On signed a-adic expansions and weakly almost periodic functions, Proc. London Math. Soc. 63 (1991), 620-656. MR 93c:43007

15. T T West, Weakly compact monothetic semigroups of operators in Banach spaces, Proc Roy Irish Acad A 67 (1968), 27-37. MR 39:824

Department of Pure Mathematics, University of Sheffield, Sheffield S3 7RH, EngLAND

E-mail address: j.pym@sheffield.ac.uk 